\title{
Active low-element antenna array for local navigation system
}

\author{
Igor Shirokov ${ }^{1, *}$, Elena Shirokova ${ }^{2}$, Igor Serdyuk $^{2}$, and Anna Mordvinova ${ }^{1}$ \\ ${ }^{1}$ Electronics Engineering Department, 299053 Sevastopol State University, Sevastopol, Russia \\ ${ }^{2}$ Radioelectronics and Telecommunications Department, 299053 Sevastopol State University, \\ Sevastopol, Russia
}

\begin{abstract}
The principles of constructing an active low-element antenna array for a local navigation system are presented. The results of calculation and modeling of a low-element lattice are presented. The selection of the parameters of the antenna array is produced. Comparative results of modeling a low-element array with passive and active antenna elements are presented.
\end{abstract}

\section{Introduction}

Currently, special attention is paid to the creation of remote traffic control systems, including the creation of crewless ships. One of the tasks when creating crewless systems is to ensure reliable navigation, especially near the coast. This task is especially relevant for creating a system of automatic mooring of a ship. The method for measuring the position of a ship proposed in [1] allows creating a local navigation system that provides the determination of the instantaneous coordinates of a ship with accuracy sufficient to provide remote control of the mooring. In this case, the main source of the error in determining the coordinates is the ship's pitching, which leads not only to a spread of coordinates due to the movement of the antenna, but also to fading of the measuring signal. Thus, to increase the distance of the positioning system, it is necessary to provide a greater signal level from the repeater and reduce the influence of multipath propagation. Due to the formation of a discretely tunable radiation pattern of the antenna array and the use of active antenna elements, the accuracy and signal to noise ratio of the local navigation system can be significantly increased.

\section{Main part}

The antenna array is an array of rectangular printed antenna elements. Elements are displaced in a plane perpendicular to the plane passing through the point of excitation of the antenna elements and their centers. Such a choice of the location of the antenna elements reduces their mutual influence on each other.

* Corresponding author: shirokov@ieee.org 
Consider a linear equidistant antenna array. The field of the entire antenna array is determined by summing the fields of individual emitters and is written in the following form [2]:

$$
E=\sum_{\mathrm{n}=1}^{\mathrm{N}} E_{\mathrm{n}}=A f_{1}(\theta, \phi) \frac{\sin \frac{N \psi}{2}}{\sin \frac{\psi}{2}} \cos \left(\omega t-k r_{0}\right),
$$

where $\psi=k d \sin \theta+\psi_{1}$ - is the phase shift between the fields of adjacent emitters at the point of observation; in this case, the multiplier $k d \sin \theta$ determines the spatial phase shift, which depends on the distance between the radiators in the array, $d$, and $\psi_{1}$ - the electrical phase shift, which is formed by phase shifters in the diagram forming device; $r_{0}$ - distance from the phase center of the antenna array to the observation point; $N$ - is the number of emitters in the linear array.

The distance between the emitters is selected according to the criterion of the absence of additional diffraction maxima according to the formula (2)

$$
\frac{d}{\lambda} \leq \frac{1}{1+\sin \theta_{0}} .
$$

To create an antenna array, a microstrip printing antenna was chosen as an array element. The advantages of this type of antenna are: simplicity of design, ease of manufacture, ease of excitation, the ability to create arrays of the same type of elements, the possibility of combining antennas, amplifiers and phase shifters in a single printing unit.

The design of a single antenna element is a square printed resonator located on the dielectric substrate metallized from the bottom. With the substrate thickness of $1.5 \mathrm{~mm}$ and the relative dielectric constant of 4.2 and the central operating frequency of $1.5 \mathrm{GHz}$, the dimensions of the antenna array element are $42 \times 42 \mathrm{~mm}$. The feed point is located at a distance of $13 \mathrm{~mm}$ from the edge of the printing element with an input impedance of $50 \mathrm{Ohms}$. The distance between the edges of the antenna elements is also $42 \mathrm{~mm}$, which corresponds to half the wavelength in the dielectric.

Table 1 shows the dependence of the width of the directional diagram and the range of scanning angles on the number of elements of the antenna array.

Table 1. Characteristics of low-element antenna arrays.

\begin{tabular}{|c|c|c|}
\hline $\begin{array}{c}\text { Number of } \\
\text { elements }\end{array}$ & $\begin{array}{c}\text { Width of radiation } \\
\text { pattern, degree }\end{array}$ & $\begin{array}{c}\text { Scanning angle range, } \\
\text { degree }\end{array}$ \\
\hline 2 & 60 & $\pm 13,5$ \\
\hline 3 & 41 & \pm 15 \\
\hline 4 & 30 & \pm 16 \\
\hline
\end{tabular}

In Fig. 1 the radiation patterns of a three-element antenna array during in-phase and non-in-phase excitation of elements are shown. Due to changes in the excitation phases of the side elements relative to the central element, one can significantly change the position of the minima of the radiation pattern, and due to this, it can reduce the effect of interference on the useful signal. 


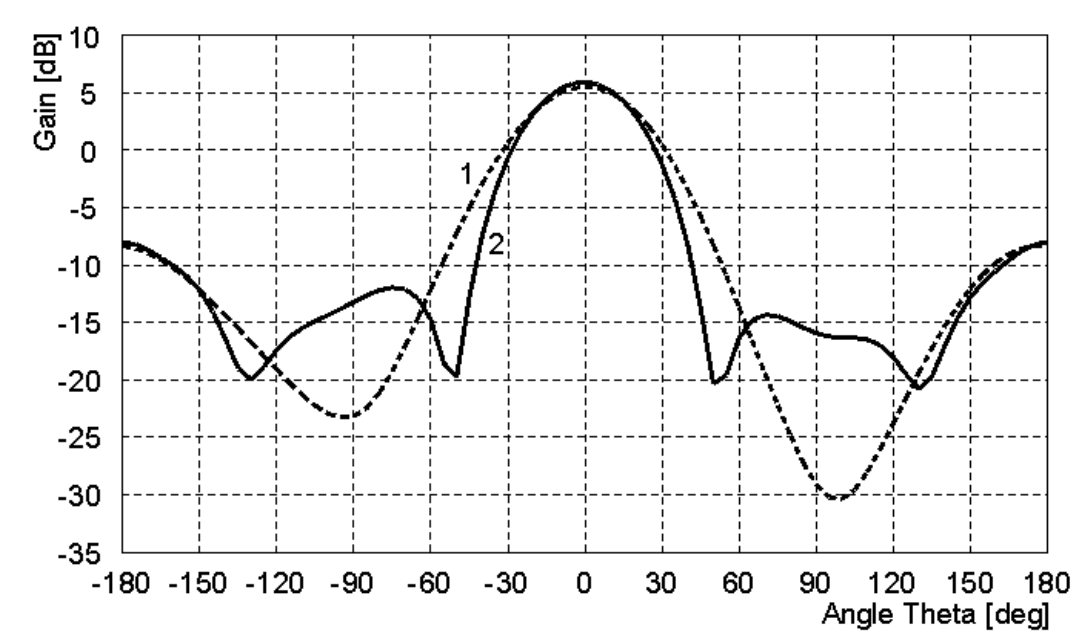

Fig. 1. Antenna pattern of a three-element antenna array (curve 1 in-phase excitation, 2 - non-in-phase excitation).

In Fig. 2 the radiation patterns of a four-element antenna array for non-phase excitation of elements and scanning of a beam in a 30-degree range are shown.

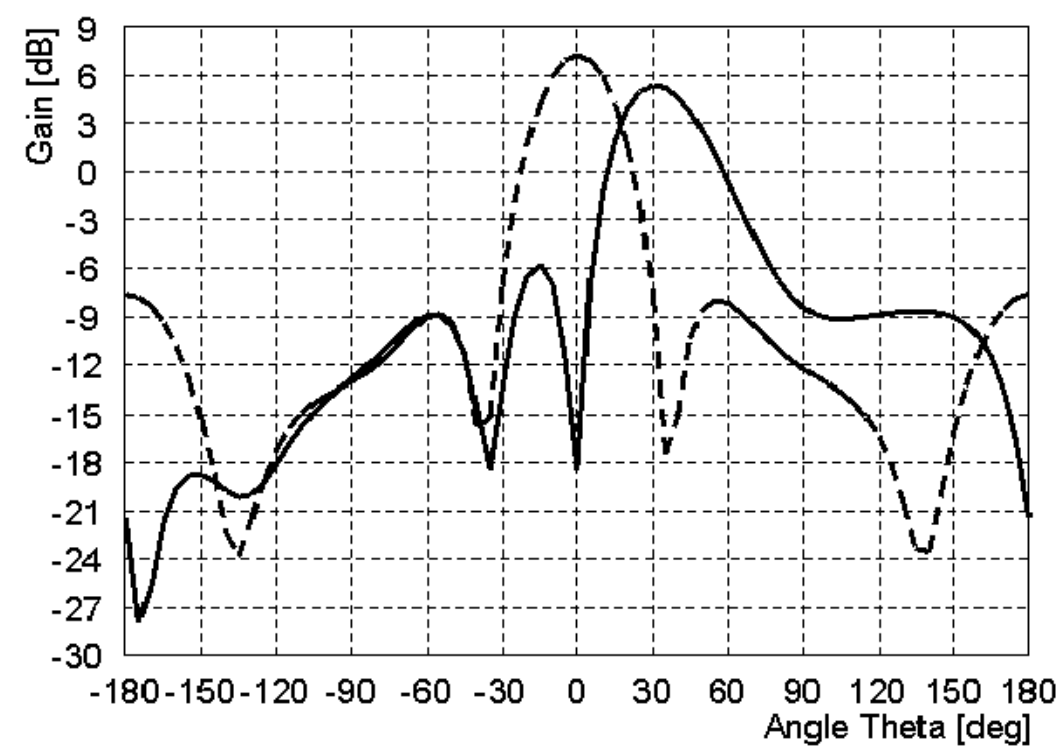

Fig. 2. Antenna pattern of the four-element antenna array.

Each element of the antenna array is combined with a single-port microwave amplifier, which makes the element of the array an active transceiver antenna [3, 4].

The microwave amplifier is realized by the scheme of single-port amplifier of Shirokov [4]. The microwave amplifier scheme is shown in the Fig. 3.

The resonant circuit in this schematic is the part of microstrip antenna. As a field-effect transistor, a double-gate field-effect transistor is used. Moreover, the first gate of the fieldeffect transistor is connected to the output of the structural inductance, and its second gate is connected to the supply terminal of the control DC voltage. By setting the required DC control voltage, the conditions for the absence of self-excitation of the circuit and signal generation are met. 


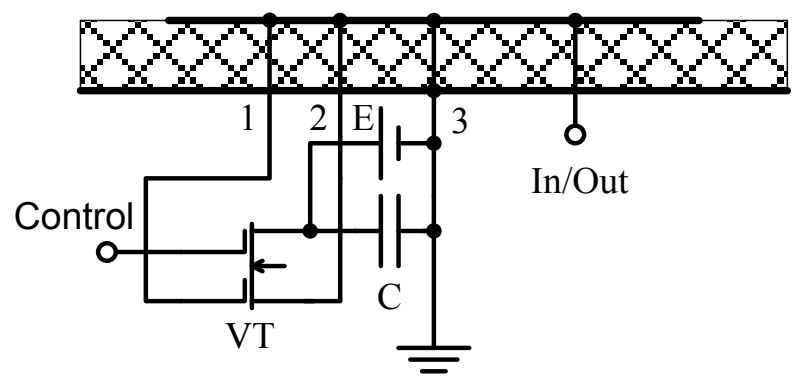

Fig. 3. The microwave amplifier schematic circuit.

A single-port resonant transistor amplifier (Fig. 3) consists of an In/Out microwave signal input and output terminal, a structural inductance with taps 1, 2, and 3, a two-gate field effect transistor VT, a blocking capacitor C, a DC voltage power supply E, and a supply terminal DC voltage Control. In this case, the structural inductance is part of the microstrip antenna.

The microwave signal through the In/Out terminal enters the microstrip antenna, the third terminal of which is connected to a common wire (Fig. 3). The first output of the constructive inductance induces an increased in amplitude microwave signal in phase with the input signal, which is fed to the first gate of a two-gate field-effect transistor. This microwave voltage causes the appearance of a common-mode current through the channel of the field-effect transistor, which partially flows through the structural inductance 2 by connecting the source of the field-effect transistor to the second terminal of the structural inductance. In this case, the current through the structural inductance 2 in its part between the first and second terminals turns out to be in phase with the input signal. Thus, positive feedback is realized and the microwave signal is amplified. The gain of the circuit reaches its maximum value at the resonance of the oscillating system formed by the structural inductance 2 and the input capacitance of the field effect transistor 1.

In the case of receiving microwave oscillations, the output amplified microwave signal is taken from the same output of the In/Out microstrip antenna [6]. In this case, the position of the In/Out terminal of the microstrip antenna is selected in terms of matching the input / output impedance of the amplifier with the impedance of the input / output feeder. The input and output microwave signals can be separated by using a directional coupler or circulator.

The blocking capacitor $\mathrm{C}$ is used to connect the drain of the $\mathrm{AC}$ field effect transistor to a common wire.

The power supply voltage of the amplifier is supplied to the drain of the field effect transistor.

In Fig. 4 the simulation results of the transmission coefficient of the microwave signal in the communication channel with the antenna element without an amplifier and with an amplifier are shown.

As it can be seen from Fig. 4 the amplifier, which is made according to this scheme, allows increasing the signal level by $10 \mathrm{~dB}$, while it is not sensitive to matching input impedance. Increasing the signal level by $10 \mathrm{~dB}$ can significantly increase the operation range of the local navigation system.

In addition, from Fig. 4 it follows that the passband of the active antenna at the $-3 \mathrm{~dB}$ level is $36.5 \mathrm{MHz}$. This is significantly less than the passband of the passive antenna $52.4 \mathrm{MHz}$, but enough for the local navigation system. The decrease in the passband of the active microstrip antenna is caused by the operation of the amplifier in resonance mode and is its inherent property. 


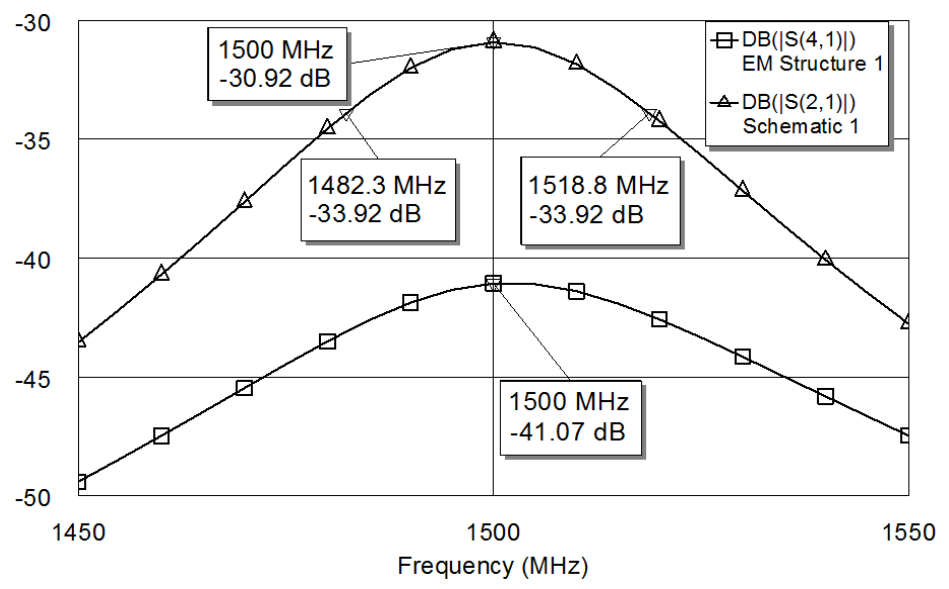

Fig. 4. The results of modeling the transmission of a microwave signal by a passive and active antenna element.

In Fig. 5 the simulation results of the VSWR dependence of antenna input port on frequency in passive and active modes are shown.

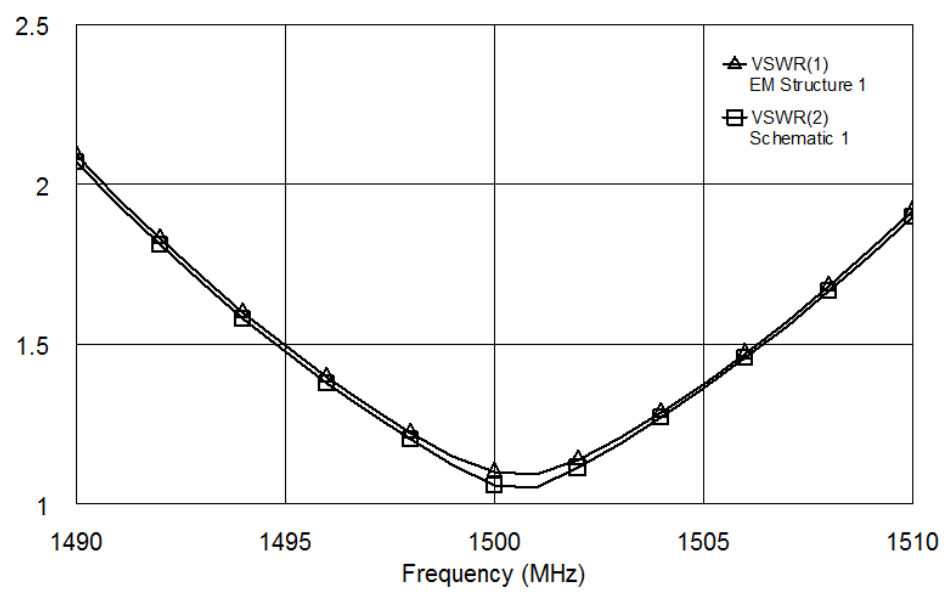

Fig. 5. The results of modeling VSWR dependence of antenna input port on frequency in passive and active modes.

As can be seen from Fig. 5 a single-port amplifier practically does not affect the VSWR of the microstrip antenna when the connection points 1 and 2 of the VT transistor are selected correctly. In this case, the antenna bandwidth at the level of VSWR equal to 2 is approximately equal to $20 \mathrm{MHz}$.

Table 1 shows the dependence of the gain on the distance between the terminals 1 and 2 of the microstrip antenna.

Table 2. The dependence of the gain on the distance between the terminals 1 and 2 of the microstrip antenna.

\begin{tabular}{|c|c|c|c|c|c|c|}
\hline $\begin{array}{c}\text { Distance between } \\
\text { the terminals } \\
1 \text { and } 2, \mathrm{~mm}\end{array}$ & 2 & 4 & 6 & 8 & 10 & 12 \\
\hline Amplification, dB & 10.88 & 11.23 & 11.47 & 11.68 & 11.93 & 11.87 \\
\hline
\end{tabular}


As can be seen from Table 1, the gain of a single-port amplifier changes within $1 \mathrm{~dB}$ when the distance between the ports changes by $1 / 8$ of the wavelength in the dielectric. Thus, the gain of a single-port amplifier is not very sensitive to the choice of the distance between ports 1 and 2. However, the VSWR of the In/Out microstrip antenna significantly depends on the location of these ports.

\section{Conclusion}

Thanks to the proposed low-element antenna array, the influence of multipath propagation of the measuring signal on the accuracy of determining the location of moving objects by the local navigation system can be significantly reduced. Moreover, an antenna array with an odd number of elements is preferable. Thus, the minimum number of elements of the antenna array should be equal to three.

Using a single-port transistor amplifier allows increasing the level of the microwave signal by $10 \mathrm{~dB}$. The advantages of this amplifier include simplicity of design and ease of setup. Moreover, the use of a single-port amplifier together with an external circulator opens up good prospects for replacing traditional two-port transistor amplifiers in local navigation and RFID systems. In addition, the use of a single-port amplifier as an active repeater eliminates directional couplers and circulators from its circuit.

\section{References}

1. R.W. McMillan, Intensity and angle-of-arrival effects on microwave propagation caused by atmospheric turbulence, IEEE Proc. of Int. Conf. on Microwaves, Communications, Antennas and Electronic Systems (COMCAS'08), Tel-Aviv, Israel, 13-14 May 2008, P. 1-10 (2008)

2. V. A. Grigoriev, S. S. Mesnac, V. L. Golusin, J. A. Raspev, O. I. Lagutenko, A. S. Mesnac, Adaptive antenna arrays, Tutorial in 2 parts, St. Petersburg, ITMO University, 179 p. (2016)

3. I. B. Shirokov, The Multitag Microwave RFID System, IEEE Transactions on Microwave Theory and Techniques, Volume 57, Issue 5, Part 2, P. 1362-1369 (2009)

4. I. B. Shirokov, The Multitag Microwave RFID System with Extended Operation Range, in book Chipless and Convention Radio Frequency Identification: Systems for Ubiquitous Tagging, Hershey PA, IGI Global, P. 197-217 (2012)

5. I. B. Shirokov, Shirokov's single-port resonant transistor amplifier, Patent \#2594335 Russia, IPC H03F 3/189, H03F 3/19, H03F 3/04, pub. 10/08/2016, Bull. \# 22

6. A. P.Venguer, J. L. Medina, R. A. Chavez, A. Velazquez, Low Noise One-Port Microwave Transistor Amplifier, Microwave and Optical Technology Letters, Vol. 33, \#3, P. 100-104 (2002) 